\title{
Metaplastic breast cancer with rapidly progressive recurrence in a young woman: case report and review of the literature
}

\author{
Jerzy W. Mituś ${ }^{1,2}$, Beata Sas-Korczyńska ${ }^{3}$, Anna Kruczak ${ }^{4}$, Marek Jasiówka ${ }^{5}$, Janusz Ryś ${ }^{4}$
}

\author{
${ }^{1}$ Department of Surgical Oncology, Centre of Oncology, Maria Skłodowska-Curie \\ Memorial Institute, Krakow, Poland \\ 2Department of Anatomy, Jagiellonian University Medical College, Krakow, Poland \\ ${ }^{3}$ Clinic of Oncology, Department of Breast and Chest Cancer, Centre of Oncology, \\ Maria Skłodowska-Curie Memorial Institute, Krakow, Poland \\ ${ }^{4}$ Department of Tumour Pathology, Centre of Oncology, Maria Skłodowska-Curie \\ Memorial Institute, Krakow, Poland \\ ${ }^{5}$ Department of Systemic and Generalized Malignancies, Centre of Oncology, \\ Maria Skłodowska-Curie Memorial Institute, Krakow, Poland
}

Submitted: 10 December 2014

Accepted: 14 February 2015

Arch Med Sci 2016; 12, 6: 1384-1388

DOI: $10.5114 /$ aoms.2016.62917

Copyright $\odot 2016$ Termedia \& Banach

Metaplastic breast carcinoma $(\mathrm{MpBC})$ is a rare neoplasia. It accounts for less than $1 \%$ of all breast carcinomas (BC), and the incidence of this type of cancer has risen since 2000 (the World Health Organization (WHO) recognized $\mathrm{MpBC}$ as a distinct type of breast cancer). It is made up of heterogeneous subgroups of malignant tumors built of neoplastic epithelium differentiated into squamous cells and/or mesenchymal-looking elements [1-4]. The most common histopathological subtypes are squamous cell carcinoma and metaplastic carcinoma with mesenchymal differentiation [5-9]. Over $90 \%$ of $M p B C S$ are ER, PR, and HER2 negative ("triple negative"), contrary to intraductal carcinomas, which are triple negative in $18 \%$ of cases [8,10-14]. The mean age of MpBC patients is about 50 years of age [7, 15-17]. In most studied cases a diagnosis may not be achieved by use of core needle biopsies, and histological examination of the surgical specimen allows for the definitive diagnosis of $M p B C$ $[9,18,19]$. There is no standard treatment regimen specific for MpBC, which is characterized by a poor prognosis. We present here a case of rapidly progressive recurrence of $\mathrm{MpBC}$.

We present a case of a 22-year-old woman, with a negative family history of BC. She came to our clinic with right breast enlargement and had experienced pain in the preceding 3 months. Physical examination showed a tumor filling the right breast, with a maximum diameter of $20 \mathrm{~cm}$. There was no skin or nipple retraction and no axillary lymphadenopathy. In ultrasound examination, the lesion appeared as a solid-liquid tumor with sharp borders, $20 \mathrm{~cm}$ in a diameter. The axillary lymph nodes were not affected. It was classified as BI-RADS category 4b. By means of a fine needle aspiration biopsy, $210 \mathrm{ml}$ of serous-bloody fluid was obtained. A core needle biopsy was performed and histological examinations showed breast tissue with non-specific, chronic inflammation around the ducts and lobules with no evidence of a neoplasm.

The patient underwent a lumpectomy during which the nipple and part of the pectoralis major muscle were removed. A histological ex-

\author{
Corresponding author: \\ Jerzy W. Mituś MD, PhD \\ Department \\ of Surgical Oncology \\ Centre of Oncology \\ Maria Skłodowska-Curie \\ Memorial Institute \\ Krakow Branch \\ 11 Garncarska St \\ 31-115 Krakow, Poland \\ Phone: +48124224928 \\ Fax: +48 124231076 \\ E-mail: \\ jerzy.mitus@gmail.com
}


amination showed macroscopically a $20 \times 18 \mathrm{~cm}$ encapsulated tumor infiltrating the muscle with central necrosis.

Microscopically, the tumor was well circumscribed and consisted of both spindle and poorly differentiated epithelial cells. The spindle cells formed long parallel fascicles (Figure 1) separated by collagen bands and deposits of amorphous extracellular material, which focally presented an osteoid-like appearance (Figure 2). The poorly differentiated epithelial cells created a solid tex-

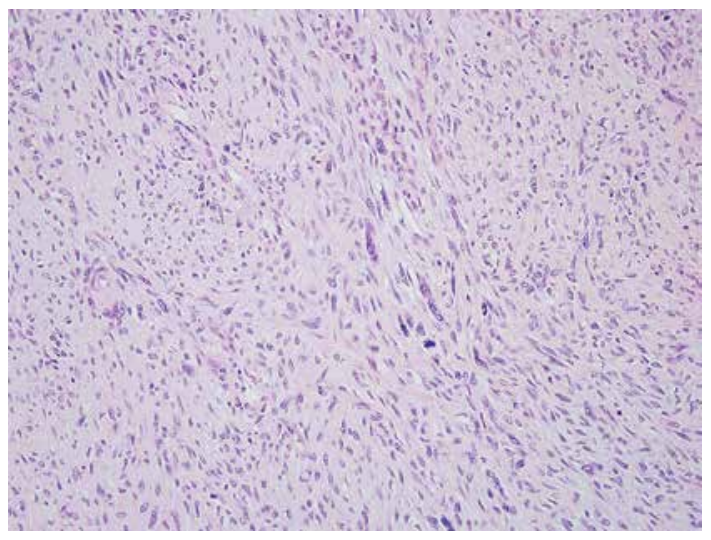

Figure 1. Spindle cell component of the tumor; high nuclear grade, numerous mitoses

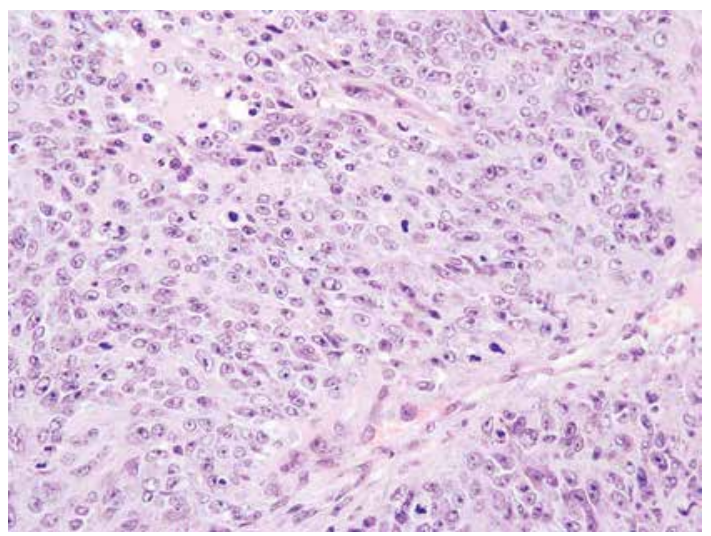

Figure 3. Poorly differentiated epithelial component of the tumor

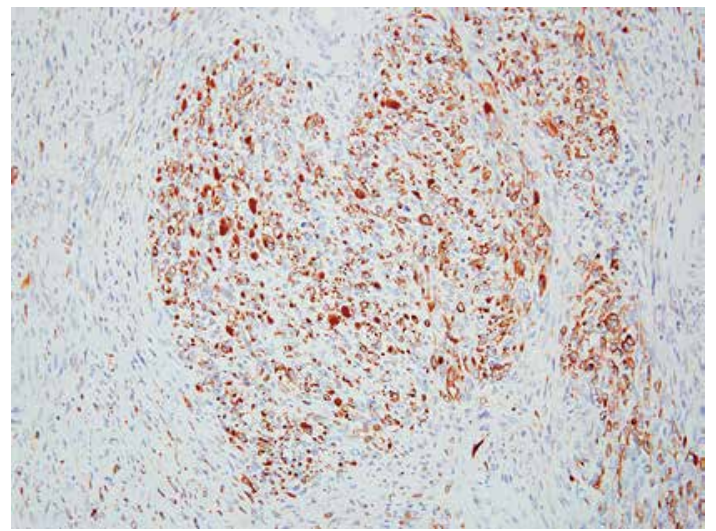

Figure 5. Positive reaction against desmin in the spindle cell component of the tumor ture (Figure 3) with extensive foci of necrosis and hemorrhages. Both spindle and epithelial cells are characterized by a high nuclear grade and high mitotic activity.

Immunohistochemical staining revealed that epithelial cells stained focally for cytokeratins CAM5.2 (Figure 4), whereas the spindle cell component reacted strongly to CD10, desmin (Figure 5), and P53 protein. Both constituents were characterized by an intensive, membranous reaction to EGFR protein (Figure 6) and were negative for

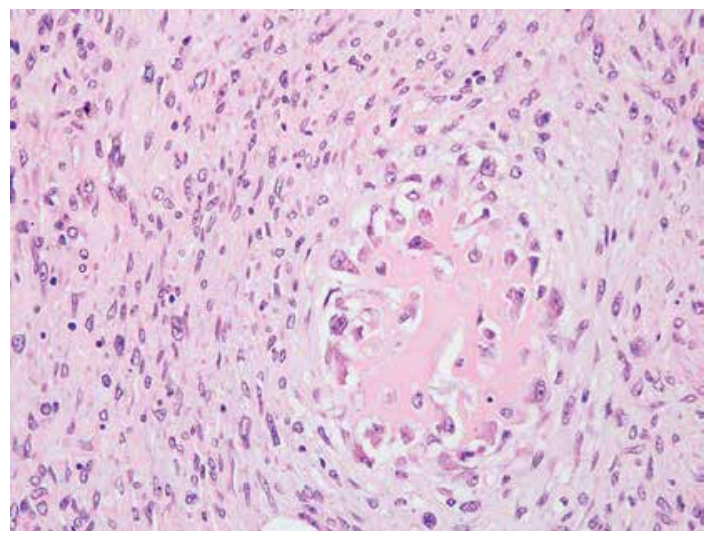

Figure 2. Osteoid formation

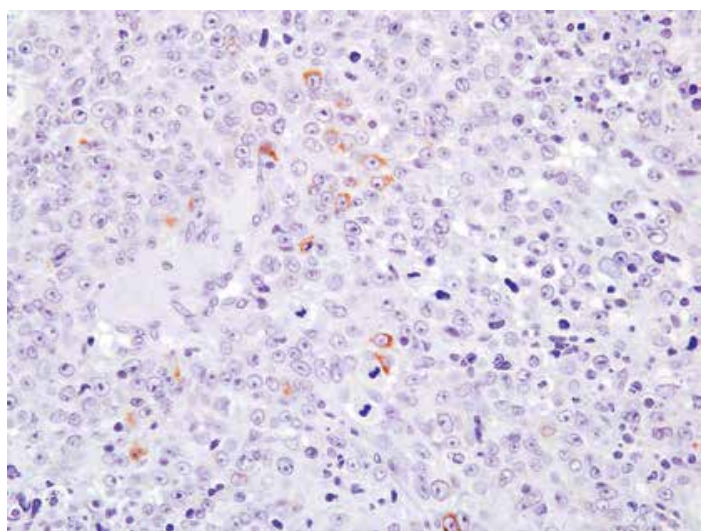

Figure 4. Anti-cytokeratin CAM5.2 positivity in the poorly differentiated epithelial tumor component

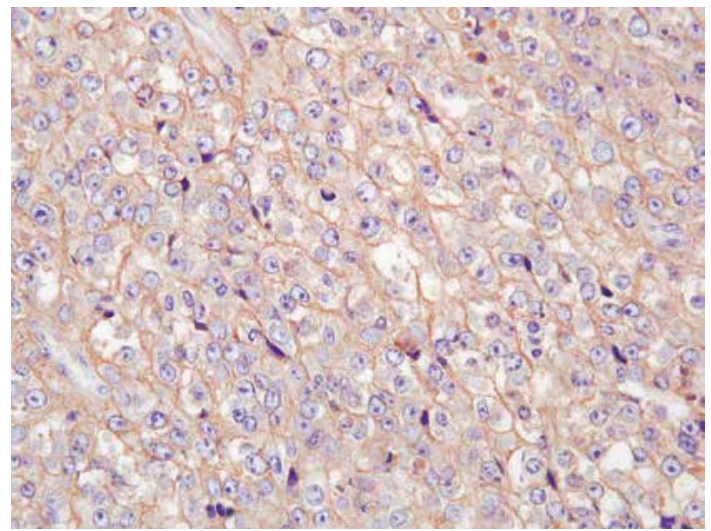

Figure 6. Diffuse membranous reaction to epidermal growth factor receptor (EGFR) 
steroid receptors (ER, PR) and HER2 protein. The $\mathrm{Ki}-67$ index ranged from $89 \%$ (in the spindle cell component) to $91 \%$ (epithelial cells). According to the WHO 2012 Classification of Tumors of the Breast, the tumor was classified as breast carcinoma with leiomyosarcomatous and osseous metaplasia (metaplastic carcinoma) high grade (G3) [1]. Margins on all sides were tumor free. The postoperative staging was assessed as pT3 pNO pMO (stage IIB).

On 39 day following the operation (22 days after the last follow-up examination), the patient came for histopathology results, and a physical examination revealed two lesions above the surgical scar. An ultrasound examination showed the lesions to be $5.9 \mathrm{~cm}$ and $4.7 \mathrm{~cm}$ in diameter. The results of the fine needle aspiration biopsy and core biopsy confirmed the recurrence of the breast tumor. The lesions had almost doubled their diameter in 10 days. A computed tomography examination (CT) showed two tumors located in the right breast. Their maximum diameters were $7.7 \mathrm{~cm}$ and $6.7 \mathrm{~cm}$. The CT scan did not show any other pathology.

The patient's tumor was excised 2 months after the primary operation followed by a Halsted reoperation of the right breast with stage I axillary lymph node dissection. A histological examination confirmed that the relapsed metaplastic breast carcinoma had been removed with a thin tumor margin diameter of $1 \mathrm{~mm}$ on the pectoral side. The lymph nodes appeared normal.

The patient was qualified for adjuvant chemotherapy comprising doxorubicin and cyclophosphamide. After the first chemotherapy cycle she received radiotherapy combined with hyperthermia. This decision was made on the grounds of dynamics of the chest wall recurrence in order to control the local process. Radiotherapy was carried out in the form of external radiation using mixed photon/electron beams generated in a linear ac- celerator. It was planned using a computer-aided therapy planning system. The beam energy was individually adjusted in consideration of the patient's anatomical parameters. The planning target volume (PTV) included the chest wall and regional lymph nodes with $1 \mathrm{~cm}$ margin. The total dose given to the PTV was 50 Gy in 25 fractions. Local hyperthermia was applied using the BSD500 system emitting electromagnetic radiation of $915 \mathrm{MHz}$ and $35 \mathrm{~W}$ in order to obtain a therapeutic temperature in the range of $40-41^{\circ} \mathrm{C}$. The patient was submitted to local hyperthermia 3 times a week (every second day) for 60 min immediately before each radiotherapy session. The area covered by hyperthermia involved the recurrence with an adequate margin. After the local treatment, the patient developed G3-skin reaction.

Following radiotherapy, chemotherapy was continued for a total of 4 cycles with good tolerance. The patient remains under clinical observation. Table I presents detailed clinical and pathological characteristics of the patient and therapeutic choices.

Recent studies show that MpBC has a worse prognosis and a higher risk of recurrence than similar stage invasive ductal carcinoma (IDC) of no special type $[11,20,21]$. Comparing patients with $M p B C$ and triple negative IDC, $M p B C$ has a lower survival rate, worse disease-free survival (DFS) (5-year DFS rate $39-56 \%$ vs. $60.3-77 \%$ ) and a worse overall survival (OS) rate (5-year OS rate $44-54.5 \%$ vs. $73.3-78 \%$ ) [2, 10, 13, 20, 22]. There are a few hypotheses that stress the pathogenesis of $\mathrm{MpBC}$, but none of them is accepted in the literature as the main cause of $\mathrm{MpBC}$, and the cellular origin of $M p B C$ is not yet clear $[3,4]$.

Our patient developed early (during 2 months) and rapidly progressive recurrence of the disease, as was confirmed by the clinical observation and the results of molecular tests. Hyperthermia was administered in combination with radiotherapy be-

Table I. Patient characteristics

\begin{tabular}{|c|c|}
\hline Clinical & 22-year old women. T3NOMO, BI-RADS 4b, $20 \mathrm{~cm}$ solid-liquid tumor, sharp borders \\
\hline Surgery & Lumpectomy (due to lack of preoperative histopatological conformation) \\
\hline Pathological & $\begin{array}{c}\text { Metaplastic carcinoma, G3 (leiomyosarcomatous and osseous metaplasia). } \\
\text { ER, PR, HER2 negative. pT3pNOpMO }\end{array}$ \\
\hline Clinical & Chest wall recurrence \\
\hline Surgery & Halsted reoperation + stage I axillary lymph nodes dissection \\
\hline Postoperative staging & pT4a pNO pMO \\
\hline Adjuvant chemotherapy & 1 course (doxorubicin and cyclophosphamide) \\
\hline Radiotherapy & $\begin{array}{l}\text { Radiotherapy (50 Gy, } 25 \text { fractions) with hyperthermia (BSD-500 system, } 915 \mathrm{MHz} \text { and } \\
\qquad 35 \text { Watt) } 3 \text { times in a week }\end{array}$ \\
\hline Adjuvant chemotherapy & 3 courses (doxorubicin and cyclophosphamide) \\
\hline
\end{tabular}


cause of previous experience with this treatment in dealing with inoperable or non-radically operable chest wall recurrence of breast cancer [23].

This variant of $B C$ is very rare, which is why only small groups of patients are presented in the literature. The rarity of the disease makes it impossible to conduct studies focused on this patient population. When comparing $\mathrm{MpBC}$ with breast adenocarcinomas, the tumor size is usually larger and the axillary lymph node metastases are less common in MpBC patients (22\% in MpBC vs. $34 \%$ in IDC) $[13,16,22]$. Hormone and HER2 receptor positivity rates are also lower in $\operatorname{MpBC}[2,13,15$, 24]. According to the literature, the cancer cells are positive for p63 in more than $90 \%$ of $\mathrm{MpBC}$, so this marker may be useful in differential diagnosis with other spindle and mesenchymal tumors [12, 25]. A Ki-67 index higher than 30\% is observed in $50 \%$ of $\mathrm{MpBC}$ cases compared with $81 \%$ observed in patients with invasive ductal carcinomas $[2,20$, 22]. EGFR overexpression is involved in $80 \%$ of $\mathrm{MpBC}$ cases, and p53 overexpression can be observed in $32-71 \%$ of cases of MpBC [26-29].

In reviewed studies lung and bone metastases were found to be more frequent than lymphatic spread [6, 17]. This behavior of $M p B C$ resembles that of sarcomas. Luini et al. reported 3 locally recurrent and 8 lung and bone metastatic patients in their $37 \mathrm{MpBC}$ series [2]. The main distant metastatic sites are the lungs, bone and the brain $[2,7,8$, 15]. The literature shows this histologic subtype of $\mathrm{BC}$ to be chemoresistant. In a high portion of cases chemotherapy is used as adjuvant systemic treatment $[15,21,24]$. However, new chemotherapeutic drugs should be considered when treating metaplastic subtypes to overcome resistance to cytotoxic agents. In addition to MpBC chemoresistance, most of them are hormone receptor negative and hormone therapy is unnecessary [18, 21, 24, 29].

In conclusion, because of the rarity of the disease and the variety of histological subtypes that exist, the opportunities for conducting studies on this patient population are limited. Therefore the role of radiotherapy and chemotherapy is not yet clearly established, and surgical treatment is often the only choice available. Hormone therapy is unnecessary. Early diagnosis and surgical treatment are crucial to achieve an optimal outcome in patients with MpBC.

\section{Conflict of interest}

The authors declare no conflict of interest.

\section{References}

1. Lakhani SR El, Schnitt SJ, Tan PH, van de Vijver MJ. WHO classification of Tumours of the Breast. IARC Lyon 2012.

2. Luini A, Aguilar M, Gatti G, et al. Metaplastic carcinoma of the breast, an unusual disease with worse prognosis: the experience of the European Institute of Oncology and review of the literature. Breast Cancer Res Treat 2007; 101: 349-53.

3. Weigelt B, Kreike B, Reis-Filho JS. Metaplastic breast carcinomas are basal-like breast cancers: a genomic profiling analysis. Breast Cancer Res Treat 2009; 117: 273-80.

4. Cooper CL, Karim RZ, Selinger C, et al. Molecular alterations in metaplastic breast carcinoma. J Clin Pathol 2013; 66: 522-8.

5. Tavassoli FA. Classification of metaplastic carcinomas of the breast. Pathol Annu 1992; 27: 89-119.

6. Wargotz ES, Norris HJ. Metaplastic carcinomas of the breast. I. Matrix-producing carcinoma. Hum Pathol 1989; 20: 628-35.

7. Esbah O, Turkoz FP, Turker I, et al. Metaplastic breast carcinoma: case series and review of the literature. Asian Pac J Cancer Prev 2012; 13: 4645-9.

8. Nowara E, Drosik A, Samborska-Plewicka M, et al. Metaplastic breast carcinomas - analysis of prognostic factors in a case series. Contemp Oncol (Pozn) 2014; 18: 116-9.

9. Jeziorski A, Bilski A, Nejc D, et al. Metaplastic cancers of the breast: classification, diagnosis, treatment and prognosis. Contemp Oncol 2004; 8: 276-79.

10. Lester TR, Hunt KK, Nayeemuddin KM, et al. Metaplastic sarcomatoid carcinoma of the breast appears more aggressive than other triple receptor-negative breast cancers. Breast Cancer Res Treat 2012; 131: 41-8.

11. Okada N, Hasebe T, Iwasaki M, et al. Metaplastic carcinoma of the breast. Hum Pathol 2010; 41: 960-70.

12. Reis-Filho JS, Milanezi F, Steele D, et al. Metaplastic breast carcinomas are basal-like tumours. Histopathology 2006; 49: 10-21.

13. Bae SY, Lee SK, Koo MY, et al. The prognoses of metaplastic breast cancer patients compared to those of triple-negative breast cancer patients. Breast Cancer Res Treat 2011; 126: 471-8.

14. Badowska-Kozakiewicz AM, Sobol M, Patera J, Kozlowski W. Immunohistochemical evaluation of human epidermal growth factor receptor 2 and estrogen and progesterone receptors in invasive breast cancer in women. Arch Med Sci 2013; 9: 466-71.

15. Rayson D, Adjei AA, Suman VJ, et al. Metaplastic breast cancer: prognosis and response to systemic therapy. Ann Oncol 1999; 10: 413-9.

16. Pezzi CM, Patel-Parekh L, Cole K, et al. Characteristics and treatment of metaplastic breast cancer: analysis of 892 cases from the National Cancer Data Base. Ann Surg Oncol 2007; 14: 166-73.

17. Oberman HA. Metaplastic carcinoma of the breast. A clinicopathologic study of 29 patients. Am J Surg Pathol 1987; 11: 918-29.

18. Rossi L, Paglicci C, Caprio G, et al. Matrix-producing carcinoma of the breast: a case report. Case Rep Oncol 2013; 6: 245-9.

19. Rungta S, Kleer CG. Metaplastic carcinomas of the breast: diagnostic challenges and new translational insights. Arch Pathol Lab Med 2012; 136: 896-900.

20. Jung SY, Kim HY, Nam BH, et al. Worse prognosis of metaplastic breast cancer patients than other patients with triple-negative breast cancer. Breast Cancer Res Treat 2010; 120: 627-37.

21. Nagao T, Kinoshita T, Hojo T, et al. The differences in the histological types of breast cancer and the response to neoadjuvant chemotherapy: the relationship between the outcome and the clinicopathological characteristics. Breast 2012; 21: 289-95. 
22. Montagna E, Maisonneuve P, Rotmensz N, et al. Heterogeneity of triple-negative breast cancer: histologic subtyping to inform the outcome. Clin Breast Cancer 2013; 13: 31-9.

23. Sas-Korczyńska B, Walasek T, Hetnał M, et al. The preliminary results and tolerance of radiotherapy in combined with hyperthermia in pilot group of patients with inoperable breast cancer localized at chest wall. Nowotwory J Oncol 2011; 61: 444-8.

24. Hennessy BT, Giordano S, Broglio K, et al. Biphasic metaplastic sarcomatoid carcinoma of the breast. Ann Oncol 2006; 17: 605-13.

25. Tse GM, Tan PH, Chaiwun B, et al. p63 is useful in the diagnosis of mammary metaplastic carcinomas. Pathology 2006; 38: 16-20.

26. Reis-Filho JS, Pinheiro C, Lambros MB, et al. EGFR amplification and lack of activating mutations in metaplastic breast carcinomas. J Pathol 2006; 209: 445-53.

27. Hennessy BT, Gonzalez-Angulo AM, Stemke-Hale K, et al. Characterization of a naturally occurring breast cancer subset enriched in epithelial-to-mesenchymal transition and stem cell characteristics. Cancer Res 2009; 69: 4116-24.

28. Lien HC, Lin CW, Mao TL, et al. p53 overexpression and mutation in metaplastic carcinoma of the breast: genetic evidence for a monoclonal origin of both the carcinomatous and the heterogeneous sarcomatous components. J Pathol 2004; 204: 131-9.

29. Beatty JD, Atwood M, Tickman R, Reiner M. Metaplastic breast cancer: clinical significance. Am J Surg 2006; 191 : 657-64. 